\title{
3D shape recognition and reconstruction based on line element geometry
}

\author{
M. Hofer, B. Odehnal, H. Pottmann, T. Steiner, J. Wallner \\ Institute of Discrete Mathematics and Geometry, TU Wien \\ \{hofer,odehnal,pottmann,tsteiner,wallner\}@geometrie.tuwien.ac.at
}

\begin{abstract}
This paper presents a new method for the recognition and reconstruction of surfaces from $3 D$ data. Line element geometry, which generalizes both line geometry and the Laguerre geometry of oriented planes, enables us to recognize a wide class of surfaces (spiral surfaces, cones, helical surfaces, rotational surfaces, cylinders, etc.) by fitting linear subspaces in an appropriate seven-dimensional image space. In combination with standard techniques such as PCA and RANSAC, line element geometry is employed to effectively perform the segmentation of complex objects according to surface type. Examples show applications in reverse engineering of CAD models and testing mathematical hypotheses concerning the exponential growth of sea shells.
\end{abstract}

\section{Introduction}

Computer Vision has adopted and extended a variety of methods from geometry (see e.g. [1, 4]). Even such a specialized field as line geometry has recently received attention in connection with generalized cameras $[14,26]$ and $3 D$ shape understanding and surface reconstruction $[3,18,17]$. The latter topic has also been addressed with Gaussian image methods [21], the extended Gaussian image [6] and the Laguerre geometry of oriented planes [15].

The present paper introduces the geometry of oriented line elements - a line element consisting of a line and a point on it. From the structural viewpoint, line element geometry is a unifying theory for the geometry of both oriented lines and oriented planes. Mainly however it is a new tool for 3D shape understanding and reconstruction capable of solving problems which previous approaches could not handle.

This paper deals with surface-like point clouds and triangle meshes. The link to line element geometry is provided by the surface normals - we assume that we can obtain a discrete number of points on the surface and estimate surface normals there (see e.g. [21]; modern 3D photography

This work was supported by the Austrian Science Fund (FWF) under grant No. S9206. and corresponding software actually deliver data points plus normals).

Previous Work. We are interested in the recognition and reconstruction of special surface types and in the segmentation of a 3D data set according to these types. Thus we limit our brief literature review to these topics. In Computer Vision, recognition and reconstruction of special shapes is often performed by methods related to the Hough transform (see e.g. [7, 10]). Pure Hough transform methods work in 'spaces of shapes' and quickly lead to high dimensions and reduced efficiency. They have therefore been augmented by geometric constructions like the Gaussian image of differential geometry [21]. Because that is constructed solely from normal vectors, it is unable to distinguish, for example, between parallel planes. Both the extended Gaussian image [6] and the geometry of oriented planes [15] are improvements and work excellent for the detection of spheres and developable surfaces. They cannot, however, easily detect rotational or helical surfaces, which in turn are handled nicely by line geometry $[3,18,17]$.

We understand all these methods as local shape detectors. Their beauty lies in the fact that after introducing appropriate coordinates for the various geometric objects associated with the original data, shape understanding and reconstruction is reduced to the simple problem of fitting linear subspaces to point cloud data. Both principal component analysis (PCA) and the RANSAC principle [4] can effectively be employed. Even if such a method does not lead to an optimal fit of the original data with a special surface (e.g. due to noise), the results are still useful for initializing nonlinear optimization procedures [21, 24].

The result of a local shape detector may be seen as a labeled image (with $N$ colors) defined on a surface. For further processing, especially surface segmentation, region growing algorithms [21] and other methods of mathematical morphology $[5,11,19,22]$ have been applied. In order to make region growing procedures stop at feature regions such as sharp edges or blended edges, Pottmann et al. [16] uses a metric which incorporates the surface normals as well. Their approach is a special case of an image manifold in the sense of Kimmel, Malladi and Sochen [9], and 


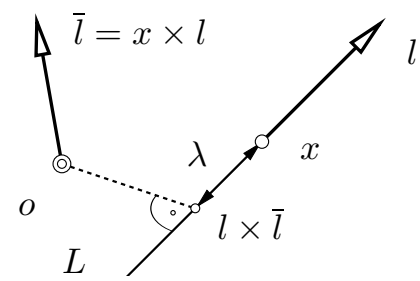

Figure 1. Coordinates $(l, \bar{l}, \lambda)$ for a line element.

is closely related to line element geometry.

Contributions of the present paper. The main contribution of the present paper is to introduce line element geometry for use in 3D shape recognition and reconstruction. Line element geometry does not appear in the classical geometric literature, despite its close relation to well studied subjects such as line geometry, Laguerre geometry, equiform kinematics [8] and spiral surfaces [25].

Our paper is organized as follows: We present the basics of line element geometry and its relation to equiform kinematics in Sec. 2. Sec. 3 shows how surface normal elements associated with the input data are used to detect a variety of special surface types. A segmentation algorithm is discussed in Sec. 4. Sec. 5 illustrates our algorithms by means of data obtained from nature and by reverse engineering of 3D technical objects.

\section{The Manifold of Line Elements}

A line element consists of a line $L$ in $\mathbb{R}^{3}$ and a point $x$ on it. We endow $L$ with an orientation, which means that we choose one of the two unit vectors parallel to $L$. Line geometry [18] coordinatizes an oriented line by normalized Plücker coordinates $(l, \bar{l})$, where $l$ is the unit vector parallel to $L$, and $\bar{l}:=x \times l$ is the momentum vector of the line. $\bar{l}$ does not depend on the choice of $x$ on $L$ and has the property that $l \cdot \bar{l}=0 . L$ is recovered from $(l, \bar{l})$ as the solution set of the 3 linear equations $\bar{l}=x \times l$. A 7-th coordinate is needed in order to locate a point $x$ on $L$ : we add $\lambda:=x \cdot l$ to the standard Plücker coordinates, thus representing a line element by $(l, \bar{l}, \lambda)$. It is elementary that $x=l \times \bar{l}+\lambda l$ (see Fig. 1). The coordinate vector $(l, \bar{l}, \lambda)$ is a point of $\mathbb{R}^{7}$. Conversely it is not difficult to show that $(l, \bar{l}, \lambda) \in \mathbb{R}^{7}$ represents a line element if and only if $l \cdot l=1$ and $l \cdot \bar{l}=0$.

Normal elements and Laguerre geometry. Each point $x$ of the surface $\Phi$ of a 3D volume has an outward unit normal vector $n$, if $\Phi$ is smooth. Thus a normal line element at $x$ is defined, whose coordinates are $(n, x \times n, x \cdot n)$. In this way a surface $\Phi$ in $\mathbb{R}^{3}$ has an associated surface $\Gamma(\Phi)$ in $\mathbb{R}^{7}$, which consists of the normal line elements of $\Phi$. We see below that for many important types of surfaces, $\Gamma(\Phi)$ lies in a linear subspace of $\mathbb{R}^{7}$. This fact is basic to surface

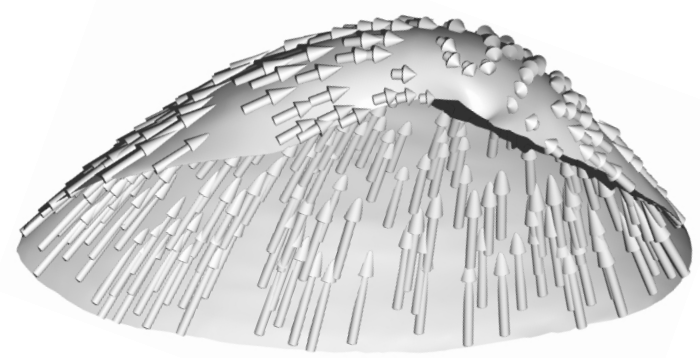

Figure 2. The velocity vector field $v(y)$ of an equiform motion according to (1), evaluated in random points $y_{i}$ of the shell of a specimen of Saxidomus nutalli. The vectors $v\left(y_{i}\right)$ are almost tangent to the surface.

recognition and reconstruction.

Other geometric objects associated with the point $x$ are the surface normal with Plücker coordinates $(n, x \times n) \in$ $\mathbb{R}^{6}$ and the oriented tangent plane with coordinates $(n, x$. $n) \in \mathbb{R}^{4}$. We see that both line geometry and the Laguerre geometry of oriented planes [15] are part of line element geometry.

Equiform motions. An equiform motion maps points $x \in \mathbb{R}^{3}$ according to $y(t)=\alpha(t) A(t) \cdot x+a(t)$, with a rotation matrix $A(t)$ and a scaling factor $\alpha(t)$. If $\alpha=$ const $=$ 1 , we obtain a rigid body motion. Like in the case of a rigid body motion, the velocity vectors $v(y)=\dot{y}(t)$ attached to the points $y(t)$ are not independent of each other and are distributed according to

$$
v(y)=c \times y+\gamma y+\bar{c} .
$$

Such a velocity vector field is visualized in Fig. 2. For our purposes it is sufficient to consider uniform equiform motions, whose velocity vector field (1) is time independent. We describe the different types of such motions and the relation between the velocity vector field (1) and their geometric characteristics. We will need the two expressions

$$
\begin{aligned}
z(c, \bar{c}, \gamma) & =\frac{1}{\gamma\left(c^{2}+\gamma^{2}\right)}\left(\gamma c \times \bar{c}-\gamma^{2} \bar{c}-(c \cdot \bar{c}) c\right) \in \mathbb{R}^{3}, \\
\mathcal{A}(c, \bar{c}, \gamma) & =\left(c, \frac{1}{c^{2}+\gamma^{2}}\left(c^{2} \bar{c}-(c \cdot \bar{c}) c+\gamma c \times \bar{c}\right)\right) \in \mathbb{R}^{6} .
\end{aligned}
$$

A uniform rigid body motion has $\alpha=1$ and $\gamma=0$. Especially a uniform translation has $A=E_{3}, c=0$ and $\dot{a}(t)$ $=\bar{c}=$ const. A uniform helical motion whose axis has the Plücker coordinates $\mathcal{A}(c, \bar{c}, 0)$ and pitch $p=(c \cdot \bar{c}) / c^{2}$ is the general case of uniform rigid body motions [18]. A uniform rotation is a special case of helical motion with $p=0$, and is recognized by $c \cdot \bar{c}=0$.

Genuinely equiform motions have $\gamma \neq 0$ and possess a center $z=z(c, \bar{c}, \gamma)$ where the velocity is zero (observed in Fig. 2). There are the central similarities with center $z$, characterized by $c=0$, and the uniform spiral motions with center $z$, axis $\mathcal{A}(c, \bar{c}, \gamma)$, and spiral parameter $p=\gamma /\|c\|:$ If 
we move $z$ into the origin and the spiral axis into the $x_{3}$-axis of a Cartesian coordinate system, a spiral motion assumes the form

$y(t)=e^{p \omega t}\left[\begin{array}{rrr}\cos \omega t & -\sin \omega t & 0 \\ \sin \omega t & \cos \omega t & 0 \\ 0 & 0 & 1\end{array}\right] \cdot x, \quad(\omega=\|c\|)$.

Surfaces generated by spiral motions are used to describe the shape of shells $[2,25]$. In Sec. 5 we test to which extent this mathematical model, which is based on exponential growth, agrees with nature.

Linear complexes of line elements. A linear complex of line elements is defined as the set of line elements whose Plücker coordinates $(l, \bar{l}, \lambda)$ satisfy the linear equation

$$
\bar{c} \cdot l+c \cdot \bar{l}+\gamma \lambda=0 \quad\left(c, \bar{c} \in \mathbb{R}^{3}, \gamma \in \mathbb{R}\right) .
$$

We call $(c, \bar{c}, \gamma) \in \mathbb{R}^{7}$ coordinate vector of the complex. A curve which undergoes a uniform equiform motion traces out an equiform kinematic surface. This concept is used by the following theorem [13] in order to show a relation between linear complexes and equiform kinematics:

Theorem 1. The surface normal elements of a regular $C^{1}$ surface in $\mathbb{R}^{3}$ are contained in a linear line element complex with coordinates $(c, \bar{c}, \gamma)$ if and only if the surface is part of an equiform kinematic surface. In that case the uniform equiform motion has the velocity vector field (1).

The shell of Fig. 2 approximates a kinematic surface very well, as the velocity vectors $v(y)$ are almost tangent to it.

\section{Classification of Surfaces}

Consider a sample $\left(l_{i}, \bar{l}_{i}, \lambda_{i}\right)(i=1,2, \ldots, N, N \geq 7)$ of surface normal elements of a surface, taken from points in general position. We want to determine if the surface is an equiform kinematic surface, or at least approximately so.

Recognizing kinematic surfaces - the exact case. We first consider data coming from an exact kinematic surface and neglect numerical issues. We compute a basis of the space of linear equations of type (4) fulfilled by all $\left(l_{i}, \bar{l}_{i}, \lambda_{i}\right)$ 's. Assume that this basis is represented by the coefficient vectors $\left(c_{1}, \bar{c}_{1}, \gamma_{1}\right), \ldots\left(c_{k}, \bar{c}_{k}, \gamma_{k}\right) \in \mathbb{R}^{7}$. Note that by Theorem 1 , each homogeneous linear equation fulfilled by the given normal element data means a uniform equiform motion which transforms the underlying kinematic surface into itself. The complete classification is given below.

- $\boldsymbol{k}=4$ : Only planes are invariant with respect to four independent uniform motions. It is trivial to find an equation of that plane from the given data.

- $\boldsymbol{k}=3$ : Spheres are invariant with respect to a threeparameter family of uniform motions (all of them rotations). The sphere's center lies on all three rotation axes with Plücker coordinates $\mathcal{A}\left(c_{i}, \bar{c}_{i}, 0\right)(i=1,2,3)$.
- $\boldsymbol{k}=2$ : (2a) A cylinder of revolution is invariant with respect to a 2-parameter family of helical motions, spanned by a translation along the helical axis, and by the rotation about that axis. So for all linear combinations $(c, \bar{c}, \gamma)=$ $\sum_{j=1}^{2} \mu_{j}\left(c_{j}, \bar{c}_{j}, \gamma_{j}\right)$ except for the one representing the translation, $\mathcal{A}(c, \bar{c}, \gamma)$ is the same, whereas the spiral center $z(c, \bar{c}, \gamma)$ is at infinity (division by zero).

(2b) A cone of revolution is invariant with respect to a 2parameter family of spiral motions, spanned by a similarity whose center is the cone's vertex, and a rotation about the cone's axis (Fig. 3.a-b). As in the cylinder case, $\mathcal{A}(c, \bar{c}, \gamma)$ is the cone's axis (except for the similarity) and $z(c, \bar{c}, \gamma)$ is the cone's vertex (except for the rotation).

(2c) A spiral cylinder, which has a logarithmic spiral as cross-section, is invariant with respect to a 2-parameter family of spiral motions with the same axis and whose spiral center varies along that axis. Translations parallel to the axis are included in this family.

- $\boldsymbol{k}=1$ : Surfaces invariant with respect to precisely one independent uniform motion (in brackets) are the following:

(1a) cylinders without rotational symmetry (translation); (1b) cones without rotational symmetry (central similarity); (1c) general surfaces with rotational symmetry (rotation); (1d) helical surfaces (helical motion); (1e) spiral surfaces (general spiral motion).

The diagram of (5) shows how to distinguish between these five cases. The axis of the surface (meaningful in cases $1 \mathrm{c}, \mathrm{d}, \mathrm{e})$ is computed by $\mathcal{A}(c, \bar{c}, \gamma)$, and its center (meaningful in cases $1 \mathrm{a}, \mathrm{b}, \mathrm{e})$ by $z(c, \bar{c}, \gamma)$.

\begin{tabular}{|c|c|c|c|c|}
\hline$c_{1}=0$ & $c_{1}=0$ & $c_{1} \neq 0$ & $c_{1} \neq 0$ & $c_{1} \neq 0$ \\
$\gamma_{1}=0$ & $\gamma_{1} \neq 0$ & $\begin{array}{c}\gamma_{1}=0 \\
c_{1} \cdot \bar{c}_{1}=0\end{array}$ & $\begin{array}{c}\gamma_{1}=0 \\
c_{1} \cdot \bar{c}_{1} \neq 0\end{array}$ & $\gamma_{1} \neq 0$ \\
\hline$(1 \mathrm{a})$ & $(1 \mathrm{~b})$ & $(1 \mathrm{c})$ & $(1 \mathrm{~d})$ & $(1 \mathrm{e})$ \\
& & &
\end{tabular}

Best fit with kinematic surfaces and PCA. Normal element data $\left(l_{i}, \bar{l}_{i}, \lambda_{i}\right)=\left(n_{i}, x_{i} \times n_{i}, x_{i} \cdot n_{i}\right)$ coming from a surface-shaped point cloud $x_{i}$ and from estimates for surface normals $n_{i}$ comprise a point cloud in $\mathbb{R}^{7}$. The coordinate system used to represent the original points $x_{i}$ is assumed to be such that $\max \left\|x_{i}\right\| \approx 1$. In order to find a linear equation with coefficient vector $(c, \bar{c}, \gamma)$ according to (4) which fits these data, we minimize

$$
F(c, \bar{c}, \gamma)=\sum_{i=1}^{N}\left(c \cdot \bar{l}_{i}+\bar{c} \cdot l_{i}+\gamma \lambda_{i}\right)^{2}
$$

under the side condition $c^{2}+\bar{c}^{2}+\gamma^{2}=1$ (which makes sense only if the point data have magnitude about 1 unit). We rewrite $F$ as $F(c, \bar{c}, \gamma)=(c, \bar{c}, \gamma) M(c, \bar{c}, \gamma)^{T}$ with

$$
M=\sum_{i=1}^{N}\left(\bar{l}_{i}, l_{i}, \lambda_{i}\right)^{T}\left(\bar{l}_{i}, l_{i}, \lambda_{i}\right) .
$$


It is straightforward that an eigenvector $(c, \bar{c}, \gamma)$ of $M$ corresponding to a numerically zero eigenvalue $\mu$ leads to an equation of type (4) which is fulfilled by the given $\left(l_{i}, \bar{l}_{i}, \lambda_{i}\right)$ 's. In that case, $F(c, \bar{c}, \gamma)=\mu$. Thus the number of small eigenvalues of $M$ (one to four) and the corresponding eigenvectors determine which type of kinematic surface the original point data are approximated with.

Classifying surfaces. When performing the classification task on a real data set, the number of decisions to be made implies that the setting of thresholds is critical. Numerical experiments have shown that the strategy described below works in a satisfactory way.

(i). Check if the original data are planar or spherical, using well known methods $[12,20]$. If they are, go to (v).

(ii). Compute $M$ and its eigenvalues and eigenvectors as described above. Sort the eigenvalues $\mu_{i}$ such that $0 \leq \mu_{1} \leq \mu_{2} \leq \ldots$. The magnitude of eigenvalues is $N$ times length squared, so use $\nu_{i}=\sqrt{\mu_{i} / N}$ for comparison purposes. As spherical and planar surfaces are excluded by now, the number $k$ of numerically zero eigenvalues of $M$ can be 0,1 or 2 . If $\nu_{1}$ is large, the best approximation of the given data by a kinematic surface is not very good, and we may choose not to proceed further (breaking up composite surfaces is the topic of Sec. 4). Otherwise, two small eigenvalues (case $k=2$ above) are detected, if $\nu_{3} / \nu_{2}>\nu_{2} / \nu_{1}$, or if both $\nu_{1}, \nu_{2}$ are smaller than a certain threshold.

(iii). In the case $k=2$, consider the eigenvectors $\left(c_{i}, \bar{c}_{i}, \gamma_{i}\right)(i=1,2)$ and compute the spiral center $z(c, \bar{c}, \gamma)$ for a number of linear combinations $(c, \bar{c}, \gamma)=\cos t\left(c_{1}, \bar{c}_{1}\right.$, $\left.\gamma_{1}\right)+\sin t\left(c_{2}, \bar{c}_{2}, \gamma_{2}\right)$ with $t=\frac{i \pi}{r}, i=1, \ldots, r, r=20$, say (Fig. 3.c). The location of these centers distinguishes between cases $2 a, b, c$. With very noisy data it is helpful to bear in mind that in real life there are no spiral cylinders if neither $2 \mathrm{a}$ nor $2 \mathrm{~b}$ fits, we might as well move on to (iv) and have the surface type detected again.

(iv). In case of one small eigenvalue, distinguish be-

(a)

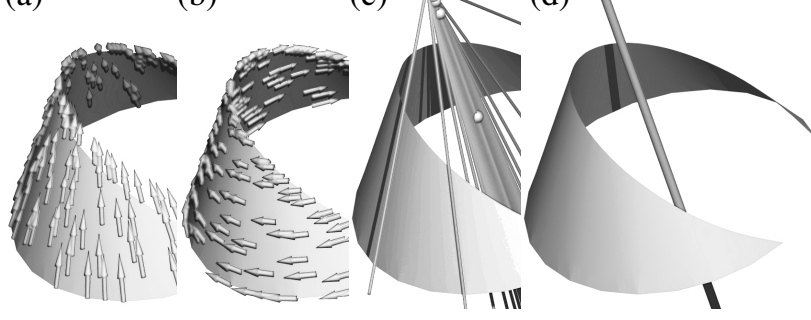

Figure 3. (a-b) Velocity vectors on a cone of revolution corresponding to eigenvectors $\left(c_{i}, \bar{c}_{i}, \gamma_{i}\right)(i=1,2)$ of $M$ via Equ. (1). (c) A sequence of 200 spiral centers and axes computed from linear combinations of these two eigenvectors. (d) Robustly reconstructed vertex and axis. tween the cases listed in (5) by choosing appropriate thresholds. These are set according to the application we have in mind and the surface types we expect. Genuine spiral surfaces are rarely observed in machine-made parts, for instance. If appropriate, compute axis and center. Finding a generator curve of the kinematic surface in question from the given point data is discussed in Sec. 5 .

(v). In all cases we get a kinematic surface $\Psi$ approximating the given point cloud. This least squares fit is made robust by iteratively downweighting outliers in the definition of $M$ :

$$
M=\frac{1}{\sum \sigma_{i}} \sum_{i=1}^{N} \sigma_{i} \cdot\left(\bar{l}_{i}, l_{i}, \lambda_{i}\right)^{T}\left(\bar{l}_{i}, l_{i}, \lambda_{i}\right),
$$

where $\sigma_{i}$ is a weight penalizing the distance of the data point $x_{i}$ from $\Psi$ and the distance of the surface normal element $\left(l_{i}, \bar{l}_{i}, \lambda_{i}\right)$ from the subspace with equation (4).

Inhomogeneous linear equations and offset surfaces. If the inhomogeneous equation $\bar{c} \cdot l_{i}+c \cdot \bar{l}_{i}+\gamma \lambda_{i}=$ $k$ with $\gamma \neq 0$ is fulfilled by normal elements $\left(l_{i}, \bar{l}_{i}, \lambda_{i}\right)$ with $\left\|l_{i}\right\|=1$, then the line elements $\left(l_{i}, \bar{l}_{i}, \lambda_{i}^{\text {new }}\right)$ with $\lambda_{i}-\lambda_{i}^{\text {new }}=k / \gamma$ fulfill the homogeneous equation (4). The change $\lambda_{i} \rightarrow \lambda_{i}^{\text {new }}$ means moving the point $x_{i}$ to $x_{i}-\frac{k}{\gamma} l_{i}$. Fortunately the new elements are normal elements of a surface again, namely of an offset at distance $k / \gamma$ of the original data. By minimizing $\widetilde{F}(c, \bar{c}, \gamma, k)=$ $\sum_{i=1}^{N}\left(\bar{c} \cdot l_{i}+c \cdot \bar{l}_{i}+\gamma \lambda_{i}-k\right)^{2}$ we can find an offset of a kinematic surface which approximates the given data, provided $\gamma$ turns out to be nonzero.

\section{Segmentation}

The bottom up approach to segmentation, where local shape detectors classify small surface patches, which are subsequently fitted together is e.g. used by N. Gelfand and L. Guibas in [3]. They employ Euclidean kinematics and line geometry in a way similar to our use of equiform kinematics and line element geometry.

We use a top-down multi-pass algorithm which first detects planes and spheres, then the cases $2 \mathrm{a}+\mathrm{b}$ of Sec. 3 , and at last the remaining cases. The description of the first pass, which uses RANSAC in the well known way together with line element geometry serves also as an introduction into the next one, where line element geometry is more prominent.

Detecting planar and spherical surfaces. The procedure described by this paragraph is standard. For a random sample of centers chosen from the original data set (denoted by $C$ ), we determine neighbourhoods $N_{i}$. Size and number of the $N_{i}$ 's are chosen in relation to the complexity of the data set: A substantial part of at least some $N_{i}$ 's should be covered by a single surface detected by the procedure. We use the RANSAC principle [4] as follows: $R$ times we choose four random points in $N_{i}$, fit a sphere $\Psi_{i j}$ to them, and find 


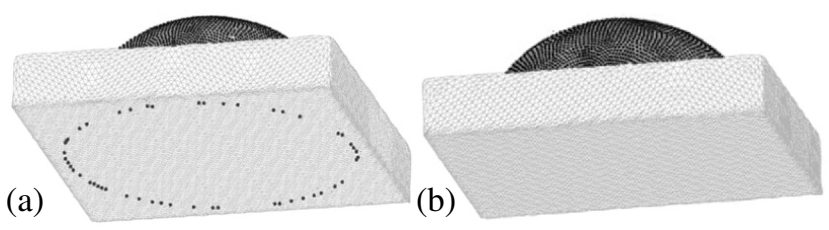

Figure 4. (a) Support $S_{i j}$ of sphere (dark) computed from point data only. (b) $S_{i j}$ trimmed using line element data.

the set $S_{i j}$ of points $x \in C$ which fulfill $\operatorname{dist}\left(x, \Psi_{i j}\right)<\delta$. For the choice of $R$ we follow [4], p. 104 and let $R=25$; for the choice of $\delta$ we take the noise level of $C$ into account. If $\Psi_{i j}$ 's radius is very large, we fit a plane instead of a sphere and recompute $S_{i j}$. $S_{i j}$ is the support of the surface $\Psi_{i j}$ (Fig. 4.a). $\Psi_{i j}$ 's with large support are investigated more closely (see next paragraph).

Trimming supports. Some vertices of $C$ close to the sphere/plane $\Psi_{i j}$ are not contained in the spherical/planar face of the given surface which we want to detect (Fig. 4.a). Their normal elements $\left(l_{r}, \bar{l}_{r}, \lambda_{r}\right)$ do not satisfy (4), where $(c, \bar{c}, \gamma)$ is any linear complex belonging to the kinematic surface $\Psi_{i j}$ according to Theorem 1 and the discussion in Sec. 3. Thus we choose exact normal line elements from $\Psi_{i j}$, compute $M$ and the eigenvectors $\left(c_{i}, \bar{c}_{i}, \gamma_{i}\right)$ for $i=1, \ldots, k$ as in Sec. 3 ( $k=3$ for a sphere and $k=4$ for a plane). A data point is kept in the support $S_{i j}$ only if its normal element satisfies

$$
\sum_{i=1}^{k}\left(c_{i} \cdot \bar{l}_{r}+\bar{c}_{i} \cdot l_{r}+\gamma_{i} \lambda_{r}\right)^{2}<\delta^{2} .
$$

See Fig. 4.b for such a trimmed support. We finally mark the support as a surface detected and iterate, until supports of spheres and planes detected become too small. This concludes pass 1 of the algorithm.

Detection of rotational cylinders and cones. Seven is the minimum number of vectors in $\mathbb{R}^{7}$ for which the question if they lie in a hyperplane makes sense. Thus for each neighbourhood $N_{i}$, we perform $R$ rounds of RANSAC as follows: We choose 7 random normal elements in $N_{i}$ to compute the matrix $M$ and its $k$ small eigenvalues and corresponding eigenvectors $\left(c_{i}, \bar{c}_{i}, \gamma_{i}\right)$ according to Sec. 3 ( $k=$ $0,1,2) .7$ degrees of freedom mean that $R \approx 100$ ensures a high probability that a kinematic surface hidden in the data is actually detected. The $j$-th round of RANSAC computes the support $S_{i j}$ as the set of line elements $\left(l_{r}, \bar{l}_{r}, \lambda_{r}\right)$ of the entire remaining data set which fulfill (9). In pass 2 of the segmentation algorithm we consider only supports where $k=2$, i.e., which lead to cylinders and cones of revolution. Fig. 5.a shows such a support consisting of all normal elements which fit the kinematics of a cylinder.

Robust computation of geometric characteristics. If a cone or cylinder " $\Psi_{i j}$ " of revolution is detected, each lin-
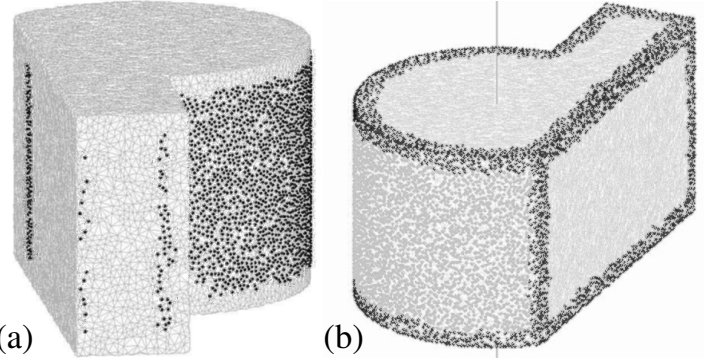

Figure 5. (a) Support of rotational cylinder computed from line element data only (dark). (b) Trimmed supports (light).
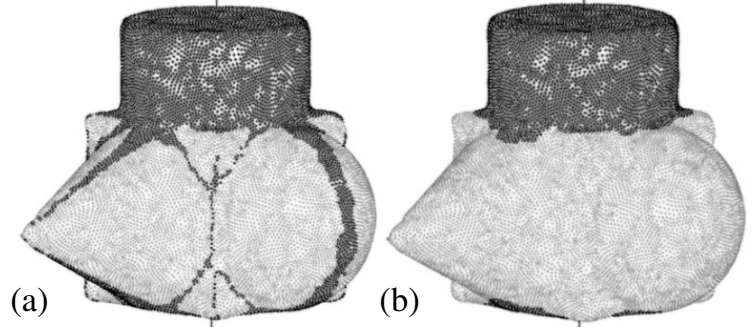

Figure 6. (a) Pass 3: data points whose normal elements fit a rotation (dark). (b) Removing curve-like parts of support.

ear combination of the first two eigenvectors according to Sec. 3 yields an axis and a center. The axes are supposed to cluster around the actual axis of $\Psi_{i j}$, and in the cone case the spiral centers cluster around the cone's vertex (Fig. 3.c). Averaging and downweighting outliers yields robustly computed geometric characteristics (axis and/or center) of $\Psi_{i j}$ (Fig. 3.d). In the cylinder case, each data point in $S_{i j}$ has a distance from the axis - we use the median of these distances as radius of $\Psi_{i j}$ and remove all points of the support too far away from $\Psi_{i j}$ (Fig. 5.b). The procedure for a cone is similar, with the aperture angle computed by a median. As in the pass 1, we mark maximal supports $S_{i j}$ as surfaces detected and iterate, until no cylinders and cones are found. The spiral cylinder (case 2c of Sec. 3) is ignored here in the unlikely case it occurs, it will be detected in pass 3 either as a general cylinder or as a spiral surface.

Simply invariant surfaces and morphological operations. Pass 3 of the algorithm repeats the procedure of pass 2 , but this time also the case $k=1$ is allowed. Because of $k=1$, (9) reduces to 1 condition. Thus the supports found will contain curve-like parts which we would like to throw away (Fig. 6.a). The opening operation of mathematical morphology $[5,11,19,22]$ can be used for that purpose. Fig. 6 shows the result of detecting a surface of revolution directly, bypassing passes 1 and 2, on an artificial data set, and subsequent opening of the support. The cleaning and closing operations remove small outliers and holes. A further enhancement is to detect and remove edges, 


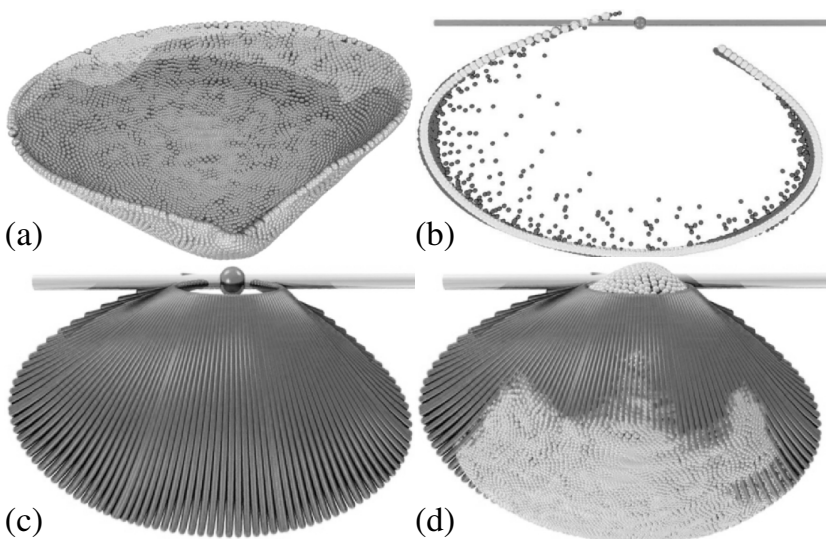

Figure 7. (a) Laser scanner data obtained from the shell of Saxidomus nutalli. (b) Axis and center of the spiral motion whose velocities are shown in Fig. 2. Data are moved into a plane and are approximated by a generator curve. (c) Kinematic surface approximating the shell. (d) combines (a),(c).

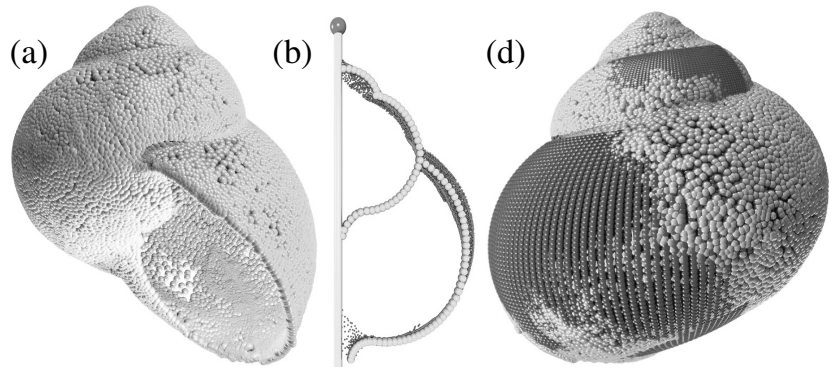

Figure 8. (a) Laser scanner data obtained from Helix pomata. (b) Axis, center and generator curve analogous to Fig. 7.b. (d) Reconstructed spiral surface and original data superimposed.

and to apply segmentation to the remaining components.

\section{Examples}

In this section we apply the procedure for fitting a kinematic surface of Sec. 3 to point data obtained by laser scanning and give examples for segmentation according to Sec. 4.

\section{Reconstructing generator curves of invariant surfaces.} The data set of Fig. 7.a is recognized as a general spiral surface by segmentation and classification algorithms. Spiral axis and center are computed from the first eigenvector of the matrix $M$ with Equ. (2).

When the given points $x_{i}$ undergo the uniform spiral motion thus detected, the spiral paths $y_{i}(t)$ are near the final kinematic surface $\Psi$. By definition, $\Psi$ is generated by subjecting a generator curve to that spiral motion. To find the generator, we select a plane through the axis and intersect the paths $y_{i}(t)$ with the plane (dark points in Fig. 7.b). If the given data really are well approximated by a spiral sur- face with the geometric parameters just computed, these intersection points must now lie in a curve-like planar point cloud. The generator is found by approximating that cloud by a curve (e.g. using the method of [23]), and $\Psi$ is complete (Fig. 7.c).

This way of finding a generator curve applies to cases 1.a-e of (5) (general cone, general cylinder, and rotational, helical, and spiral surface). It is a common feature of these surface classes that a member of the class cannot be described by a finite number of parameters like in the case of multiply invariant surfaces $(k>1$ in Sec. 3$)$, but is generated by the motion of an arbitrary curve.

Downweighting outliers yields a better approximant: We may let $\sigma_{i}=1 /\left(1+C \delta_{i}^{2}\right)$ in (8) and approximate again. Fig. 8 illustrates the same procudure with a land snail's shell. For shape analysis of shells, it is interesting to compute spiral axes and centers for various subsets of a given data set, as illustrated in Fig. 9. They turn out to be contained in the transparent cylinder and sphere which are shown in Fig. 9 and which represent axis and center of a spiral surface fitted to the entire data set.

Segmentation examples. Point clouds (16K and $50 \mathrm{~K}$ vertices) obtained by laser scanning the object shown in Fig. 10.a undergo the segmentation algorithm. Pass 1 for instance, detectes planes (Fig. 10.b). We want to know if the object happens to possess simpler parts which together make up a surface invariant only with respect to one equiform motion: We apply pass 3 of segmentation to the entire data set and get the result shown by Fig. 10.d - a large part of the object is a general cylinder and thus translationally invariant. Small parts of the surface which do not fit this cylinder, like the protruding features visible in the lower part of the object, are recognized only at the higher resolution (Fig. 10.c).

\section{Conclusions and Future Research}

We showed how line element geometry and equiform kinematics can be employed in constructing local shape detectors for surfaces and a top-down segmentation method. The surfaces handled by this approach include simple shapes, but also equiform kinematic surfaces in general, which are general cylinders (translation-invariant surfaces), general cones (homothetically invariant surfaces), and rotational, helical, and spiral surfaces. Robustness is achieved with standard techniques like RANSAC. We showed reverse engineering as well as zoology applications. The fact that surface normals are used in a prominent way implies a direction of future research: the connection with the feature sensitive metric [16] and its applications. 


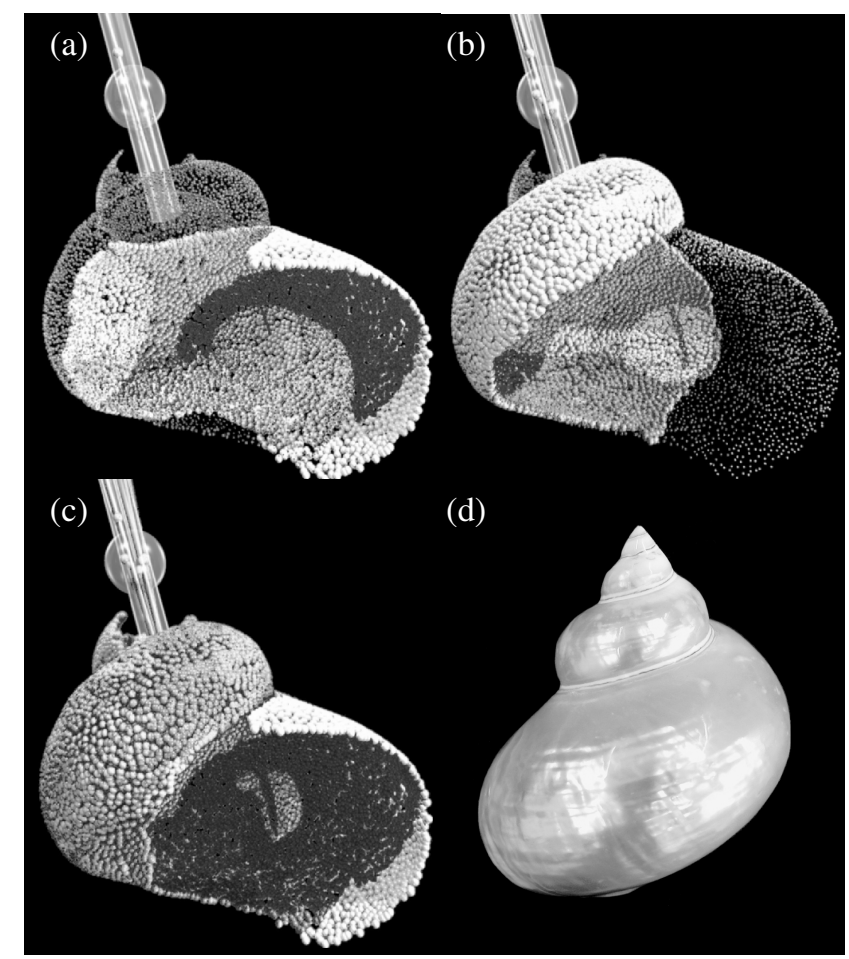

Figure 9. (a)-(b) Laser scanner data of Turbo marmoratus and reconstruction of spiral axis from different parts of the surface. (c) the same for the entire surface. (d) Picture of the shell.

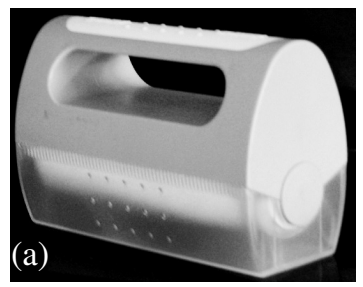

(b)
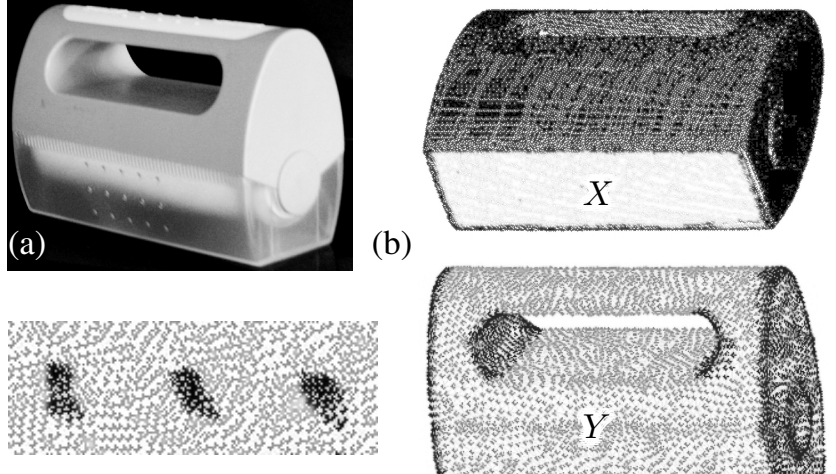

(c)

(d)

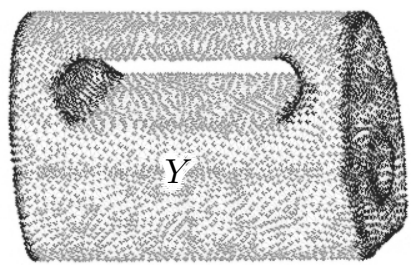

Figure 10. (a) Picture of original object. (b) Applying pass 1 of the segmentation algorithm to a $50 \mathrm{~K}$ point cloud: a planar face " $\boldsymbol{X}$ " is detected. (c) Detail (see text). (d) Applying pass 3 of the segmentation algorithm to $16 \mathrm{~K}$ point cloud: a general cylinder " $Y$ " is found.

\section{References}

[1] O. Faugeras. Three-dimensional Computer Vision: A Geometric Viewpoint. MIT Press, 1993.

[2] D. Fowler, H. Meinhardt, and P. P. Modeling seashells. In Proc. SIGGRAPH, pages 379-387, 1992.
[3] N. Gelfand and L. Guibas. Shape segmentation using local slippage analysis. In R. Scopigno and D. Zorin, editors, Proc. Symp. Geom. Processing 2004, pages 219-228.

[4] R. Hartley and A. Zisserman. Multiple View Geometry in Computer Vision. Cambridge Univ. Press, 2000.

[5] H. Heijmans. Morphological Image Operators. 1994.

[6] B. Horn. Extended gaussian images. Proc. of the IEEE, 72:1671-1686, 1984.

[7] J. Illingworth and J. Kittler. A survey of the Hough transform. Computer Vision, Graphics and Image Processing, 44:87-116, 1988.

[8] A. Karger and J. Novák. Space kinematics and Lie groups. Gordon and Breach, 1985.

[9] R. Kimmel, R. Malladi, and N. Sochen. Images as embedded maps and minimal surfaces: movies, color, texture and volumetric medical images. IJCV , 3:111-129, 2000.

[10] V. Leavers. Which Hough transform? CVGIP: Image Underst., 58:250-264, 1993.

[11] N. Loménie, L. Gallo, N. Cambou, and G. Stamon. Morphological operations on delaunay triangulations. In Int. Conf. Pattern Recognition 2000, volume 3, pages 3556-3560.

[12] G. Lukács, R. Martin, and A. Marshall. Faithful leastsquares fitting of spheres, cylinders, cones and tori for reliable segmentation. Proc. ECCV '98, 1:671-686.

[13] B. Odehnal, H. Pottmann, and J. Wallner. Equiform kinematics and the geometry of line elements. Technical Report 128, Geometry preprint series, TU Wien, 2004.

[14] T. Pajdla. Stereo geometry of non-central cameras. PhD thesis, CVUT Praha, 2002.

[15] M. Peternell. Developable surface fitting to point clouds. Comp. Aided Geom. Design, 21:785-803, 2004.

[16] H. Pottmann et al. The isophotic metric and its application to feature sensitive morphology on surfaces. In Proc. ECCV 2004, volume IV, pages 560-572.

[17] H. Pottmann, M. Hofer, B. Odehnal, and J. Wallner. Line geometry for 3D shape understanding and reconstruction. In Proc. ECCV 2004, volume I, pages 297-309.

[18] H. Pottmann and J. Wallner. Computational Line geometry. Springer, 2001.

[19] C. Rössl, L. Kobbelt, and H.-P. Seidel. Extraction of feature lines on triangulated surfaces using morphological operators. In Proc. Smart Graphics 2000. AAAI Press.

[20] T. Várady, T. Benkő, and G. Kós. Reverse engineering regular objects: simple segmentation and surface fitting procedures. Int. J. Shape Modeling, 4:127-141, 1998.

[21] T. Várady and R. Martin. Reverse engineering. In Handbook of CAGD, pages 651-681. Elsevier, 2002.

[22] J. Verly and R. Delanoy. Adaptive mathematical morphology for range imagery. IEEE Trans. Img. Proc., 2:272-275, 1993.

[23] W. Wang, H. Pottmann, and Y. Liu. Using squared distance minimization for curve reconstruction from unorganized data points. Technical Report TR-2004-11 (CS series), University of Hong Kong, 2004.

[24] A. Willis, X. Orriols, and D. Cooper. Accurately estimating sherd 3D surface geometry with application to pot reconstruction. In Proc. CVPR'03, Vol. 1, page 5. IEEE press.

[25] W. Wunderlich. Darstellende Geometrie, volume 2. 1967.

[26] J. Yu and L. McMillan. General linear cameras. In Proc. ECCV 2004, volume II, pages 14-27. 